Volume 10, No.3, May - June 2021

International Journal of Advanced Trends in Computer Science and Engineering

Available Online at http://www.warse.org/IJATCSE/static/pdf/file/ijatcse581032021.pdf

https://doi.org/10.30534/ijatcse/2021/581032021

\title{
A Smart Deforestation Monitoring and Control System Model
}

\author{
Ibam E. O. ${ }^{1}$, Olowokere V. ${ }^{2}$ \\ ${ }^{1}$ Department of Information Systems, Federal University of Technology Akure, Nigeria, eoibam@ futa.edu.ng \\ ${ }^{2}$ Adekunle Ajasin University Akungba-Akoko, Nigeria, olowokerevic@gmail.com
}

\begin{abstract}
One of the greatest challenges facing effective forest management in the world is the increasing rate of illegal logging and encroachment. This is more rampant in the tropical rainforest ecosystem of Nigeria due to its richness in desirable tropical hardwood timber species and fertile land. Government policies, institutional support in forest management and enlightenment have not succeeded in eliminating this menace. This situation presents real challenges to professionals in forest management that it has become difficult to determine the future of tropical rainforest ecosystem in Nigeria and other developing countries due to the negative impacts of illegal logging include increased incidences of global warming, environmental degradation, biodiversity, loss of revenue to government and so on. Therefore, the need to develop effective intelligent strategies or solutions to combat the menace has become inevitable. This work presents the Model design and implementation of a Deforestation Control and Monitoring System with an intelligent framework for deforestation detection and control system using machine learning algorithm and wireless sensor network, that take proactive and reactive measures to curb deforestation. The proposed system consists of four layers the physical layer, the communication layer, Knowledge layer, and presentation layer. The system is developed in an environment characterized by Unity 3D incorporated with C\# as the front end and MySQL as the backend. Unity 3D simulation tool is used for the experimental test bed and python is used for image processing and classification.
\end{abstract}

Key words : Deforestation Detection and Control System, Global warming, Network Framework, Wireless Sensor Network.

\section{INTRODUCTION}

There is increasing rate of illegal logging and encroachment all over the world. This situation is more visible in the tropical rainforest ecosystem of Nigeria because of its richness in desirable tropical hardwood timber species and fertile land.
Government policies, institutional support in forest management and enlightenment have not succeeded in totally eliminating this menace. This situation has been presenting real challenges to professionals in forest management because it is now very difficult to determine the future of the tropical rainforest ecosystem in Nigeria and other developing countries. Generally, illegal logging is the indiscriminate removal of logs from the forest without following the due process highlighted in government's logging and harvesting policy [1]. Today, Illegally harvested timber constitutes more than $50 \%$ of the value of timber harvested in the countries like Nigeria.

Effects of illegal logging and encroachment includes environmental degradation and landscape deterioration, loss of plant and animal species (biodiversity) and their potential importance to man, loss of revenue to the government, global warming, loss of economic species like 'Alchornea cordifolia' used for medical plant, 'Anthonotha macrophylla' the wood used for telegraph poles e.t.c. Tropical deforestation accounts for about 15 percent of the world's global warming pollution-more than that produced by every car, truck, plane, ship, and train on earth [2]. This explains how tropical deforestation contributes to global warming, and how actions to protect tropical forests should be put in place. Global warming is caused by a variety of gases and materials in the atmosphere; including huge amounts of carbon dioxide $\left(\mathrm{CO}_{2}\right)$ and methane $\left(\mathrm{CH}_{4}\right)$ from human activities such as extracting and burning fossil fuels, and clearing forests. These gases can trap heat in the atmosphere, causing steadily increasing temperatures. Tropical forest trees like all green plants, takes in $\mathrm{CO}_{2}$ and release oxygen during photosynthesis. During respiration they emit carbon dioxide, but in generally smaller amounts than what they take in during photosynthesis. The remaining carbon is stored in the tree, allowing it to grow bigger. That stored carbon is released into the air as carbon dioxide if the tree is burned or cut down and allowed to rot. To protect tropical forests, carbon emissions must be reduced to the levels needed to avoid the most dangerous and expensive global warming impacts.

However, surveillance plays an important role in forest management. It had been used in the past and is still being used for monitoring and information collection. Ground-based techniques generally include surveillance by on-site security staff and mobile patrols monitoring the property by water, land and air [3]. Nowadays, other 
Ibam E. O. et al., International Journal of Advanced Trends in Computer Science and Engineering, 10(3), May - June 2021, 1901 - 1913

technological methods are used including: aerial photographs, automatic video surveillance, wireless surveillance systems, Wireless Sensor Network (WSN) and satellite imagery.

Remote sensing technologies are increasingly used to monitor landscape change in many parts of the world. While the availability of extensive and timely imagery from various satellite sensors can aid in identifying the rates and patterns of deforestation, modeling techniques can evaluate the socioeconomic and biophysical forces driving deforestation processes. From a technical perspective, a sensor is a device that translates parameters or events in the physical world into signals that can be measured and analyzed. Another commonly used term is transducer, which is often used to describe a device that converts energy from one form into another. A sensor, then, is a type of transducer that converts energy in the physical world into electrical energy that can be passed to a computing system or controller.

Nowadays, portable consumer hardware, such as notebooks, mobile phones, and digital photo cameras are allowing us to create videos, while faster internet access and growing storage capacities enable us to publish directly or share videos with others. However, even though the importance of video data is increasing, the possibilities to analyze it in an automated fashion are still limited. In any surveillance application the first step is detection and tracking the human or interested object [4]

One of the major applications of wireless sensor networks is in event detection where a sensor network is monitoring certain phenomenon and the respective communication node needs to get triggered on occurrence of a certain event. Subsequently the event needs to be reported to the sink node as quickly as possible. The communication nodes can be sleeping for most of the time to conserve power since most of the events are rare in nature. There must be a mechanism to wake them up for quick event transmission through appropriate synchronization. Some of the prominent applications of this category are detection of fire, intrusion, earth quake, landslide, theft of assets and other surveillance applications [5].

WSN system design for forest monitoring involves:

(i)Sensors

(ii) Design of low power wireless communication module

(iii) Simulation and implementation of energy efficient protocol

(iv) Deployment strategies

(v) Middleware

Sensors link the physical with the digital world by capturing and revealing real-world phenomena and converting these into a form that can be processed, stored, and acted upon. Integrated into numerous devices, machines, and environments, sensors provide a tremendous societal benefit. They can help to avoid catastrophic infrastructure failures, conserve precious natural resources, increase productivity, enhance security, and enable new applications such as context-aware systems and smart home technologies [6].

To carry out adequate surveillance of the physical world like a forest area, a control system which is an interconnection of components forming a system configuration that will provide a desired system response will be highly desirable.

This research is therefore motivated by the fact that the effects of deforestation on human existence such as global warming, environmental degradation, biodiversity, loss of revenue to government, for the past few decades have been of great concern to individuals, researchers and even governmental bodies in general. Though there are many types of techniques developed for deforestation detection but they contain little corresponding real time technical measures put in place in regards to its control.

\section{RELATED WORKS}

[7] proposed A Wireless Sensor Network Based Habitat Monitoring for Protection of Endangered Species. This approach presented a model which gives a live streaming of the events monitored that employs a Passive InfraRed (PIR) based human detection. When the human presence is felt, the system detects and conveys the message over the Internet to the web page. The droughts in the extreme summer area and forest fire are monitored by constantly keeping an account of the temperature. The temperature variation is analyzed for the higher increase in temperature and assessed for the forest fire or drought. All the information sensed by the proposed system is communicated to the forest officials which could be viewed from anywhere in the world. However, this work fails to differentiate between forest officials gaining access to the forest and the deforesters before sending message(s) to the web page.

[8] proposed an Intruder Detection and Forest Fire Alert System Using Wireless Sensor Network (WSN).

The research modeled:

(i) A design for intruder detection which is made up of stand-alone boxes, with each box consisting of various solar powered sensors like humidity and temperature sensors. These boxes are spread around the entire forest area so that a complete coverage is obtained. These sensors being the most significant factor in determining the efficiency of the entire system collect the data wirelessly and transmit the data to a base station. The sensors form a cluster and are active always. The topology for intruder detection is done using the laser beams.

The network is deployed at the boundary of the forest only as the detection needs to be done only at the edges of the forest. The entry of humans into the restricted areas of the forest as well as exit of wild animals from the forest can be detected.

(ii) A design for early fire detection based on humidity and temperature change. Thus if the humidity of the air is below a threshold value and the temperature is higher than the threshold value then an alert signal is sent to the control center. However, the limitation to this approach is that the system will not be reliable during the raining periods due to 
little or total absence of light intensity to the solar panel deployed to power the system.

[9] proposed an Energy-Efficient WSN Architecture for Illegal Deforestation Detection.

The authors presented Forest Guardian WSN which collects acoustic data in order to detect the sound signature of deforestation activities, e.g. chain-saw noise. The sensors are positioned on selected trees to permit nodes to overlap in their sensing and communication ranges. Each sensor node identifies their position and spatial orientation. These nodes have the capability of localizing the source of deforestation activity within their operating range, relative to their own position, in order to determine if the deforestation occurs within a restricted zone. If so, nodes must provide to the authorities the coordinates of the suspicious activity, converting the relative position of the deforestation noise to absolute Global Position System (GPS) coordinates. This in turn depends on the SN's capability of localizing themselves on the ground. In this monitoring scenario, SN's are responsible with the detection of logging activity within their range, discriminating from other noises. However, due to long range distance between the Forest Guardian WSN router and the WSN user, degradation may be a limiting factor.

[10] proposed Amazing Amazon: Detecting Deforestation in our Largest Rainforest. The research was motivated by the fact that deforestation is an urgent problem in our world today, as it contributes to reduced biodiversity, habitat loss, climate change, and other devastating effects. The objective is to tackle the problem of deforestation using satellite data to track deforestation and help researchers to better understand where, how and why deforestation happens, and how to respond to it. The author gave a preliminary analysis of the distribution of labels in a dataset to investigate whether certain labels tend to co-appear in images using the equations (1) and (2).

$$
\begin{aligned}
& p_{x}=1 / \sum_{i=1}^{n} x \\
& p_{x \backslash y}=\frac{\sum{ }_{i=1}^{n} x_{i} y_{i}}{\sum{ }_{i=1}^{n} y_{i}}
\end{aligned}
$$

Where $n=$ no of images, $x$ and $y$ are two set of labels, $\mathrm{P}_{x}=$ the proportion of images that contain label $x$, and $\mathrm{P}_{x / y}$ be the proportion of images that contain label $x$ given that label $y$ is present. The change in appearance of label $x$ in the presence of $y$ is given by equation (3)

$$
C_{x y}=\frac{p_{x \backslash y}}{p_{x}}=\frac{n \sum_{i=1}^{n} x_{i} y_{i}}{\sum_{i=1}^{n} y_{i} \sum_{i=1}^{n} x_{i}}
$$

The work was able to show that Convolutional Nets are suitable approach for classifying land use and atmospheric conditions in the Amazon Rainforest, and are able to perform well in a multi-label setting. However, the limitation is that the challenges faced by these methods are the limited effectiveness in detecting small-scale deforestation or differentiating between human causes of forest loss and natural causes.

In [11], a Mobile Embedded Wireless Sensor Network to Prevent Deforestation was carried out. The work is motivated by the fact that deforestation is a main environmental concern in the world which may be the first link in a chain of environmental degradation that includes erosion, climatic changes, loss of biodiversity in the forest environment. The main objective is to develop an embedded based forest monitoring robot to monitor temperature, earth vibrations, landslides, tree falling, etc., in the forest area based on event detection. The authors, among the three main wireless standards (which includes Bluetooth, Zigbee and Wi-Fi), available to monitor forest environment adopted the Zigbee standard. A robot is fitted with necessary sensors and driven by battery powered motor to roam the forest area in fixed routes or through guided routes. The sensors will keep on monitoring the various parameters like temperature, vibration etc. In the event of any abnormality a wake-up signal (an interrupt signal) is generated to initiate the processor to record and transmit the information to central station, through wireless system. The wake-up signal can be generated by comparing the generated signal with a predetermined set value using Operational Amplifier (op-amp) based comparators. An H-bridge motor driver is used for the forward and reverse movement of the robot. As shown in Figure 2.1, the WSN system periodically acquires sound samples (e.g chainsaw devices) from the environment, processes it and transmits it to the central server. There is a characteristic sound associated with chainsaw during a logging activity. Whenever, the sound samples acquired from the sensors matches the sound samples of logging tools, a wood logging activity is detected and the concerned personnel is notified through an SMS alert or e-mail generated using wireless GSM or GPRS technology.

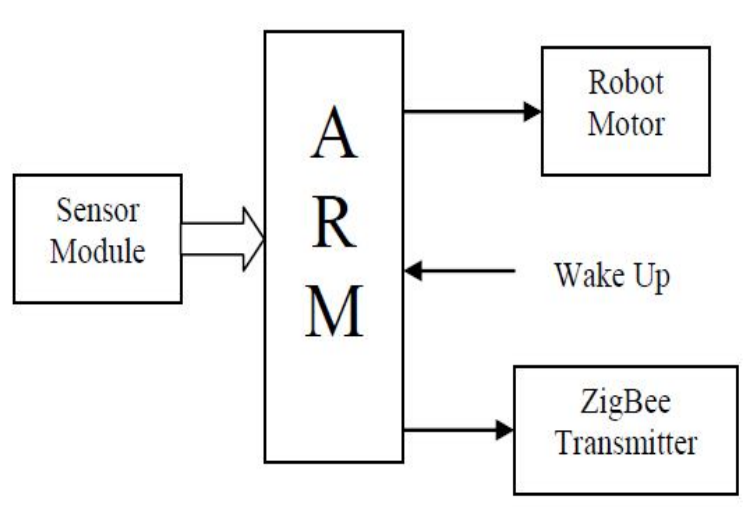

Figure 2.1: Monitoring station [11] 
Ibam E. O. et al., International Journal of Advanced Trends in Computer Science and Engineering, 10(3), May - June 2021, 1901 - 1913

The work was able to provide a forest monitoring system implemented by Advance RISC (Reduced Instruction Set Computer) Machine (ARM) controller and the sensor network based on the event detection. However, the main drawback of this system is that it works within a customized forest environment and also consumes more power because it transmits all information to control station without any event occurrence.

[12] proposed Innovative Protection of Valuable Trees from Smuggling Using RFID and Sensors. The research is motivated by efforts the efforts of different researchers that have adopted various techniques such as Zigbee WSN, sound techniques, laser beam method, e.t.c to detect deforesters, encroachers, smugglers into restricted forest areas around the world. Another technique such as the Radio Frequency Identification (RFID) in conjunction with these existing techniques will also provide the best approach in solving deforestation challenges which has negatively affected the world ecosystem and biodiversity. The objective is to provide a robust and realistic technology for protection of valuable trees such as Sandal wood, Teak wood and Rosewood from smuggling using RFID, ZIGBEE, GPS, GSM and sensors. The work presented a technique in which an RFID is fitted in each tree to serve as a unique identification. Forest officer would be holding the tracking device, in which RFID reader is embedded as he moves through the forest. Once within the reader coverage area, the reader will detect the tags. The reader would send an encoded radio frequency signal to the tag embedded in the tree, and thus power sourcing the tag by exciting the current in the antenna of the tag. The tag would respond by reflecting and modulating back its identification information including the unique number identifying the tree. The same information (the tag identifier) would be updated in the database of the workstation. The vibration sensor is used to detect the vibration level of tree. If any person tries to cut the particular tree, then the vibration level of tree will be high. The vibration sensor triggers the microcontroller while its value exceeds its threshold value. Continuity checker is used to link all the trees in a network. The research suggests protection of valuable trees from smuggling using RFID, ZigBee and various sensors. However, this work fails in the following areas:

(a) Forest officer should walk through the forest all the time.

(b) Maintaining database is such a crucial process.

(c) The system cannot find tree logging before it is cut down fully.

The general observation is that the above reviewed related works can to some extent provide reactive measures, which at that time the logging has taken place and the side effect of deforestation already activated but lack proactive measures which is very essential to initiate processes capable of preventing the cutting down of the trees in the first place. Therefore, our approach will provide the proactive and reactive approaches using intelligent techniques and cost effective technologies.

\section{SYSTEM DESIGN}

The proposed system involves a design of an intelligent framework for deforestation detection and control system using machine learning algorithm and wireless sensor network for proactive and reactive measure. It consists of four (4) layers namely; the physical layer (flex sensor, micro-controller, and IP camera), the communication layer (internet and LoRaWAN), Knowledge layer (substation sever and database), and presentation layer (webserver and mobile devices).

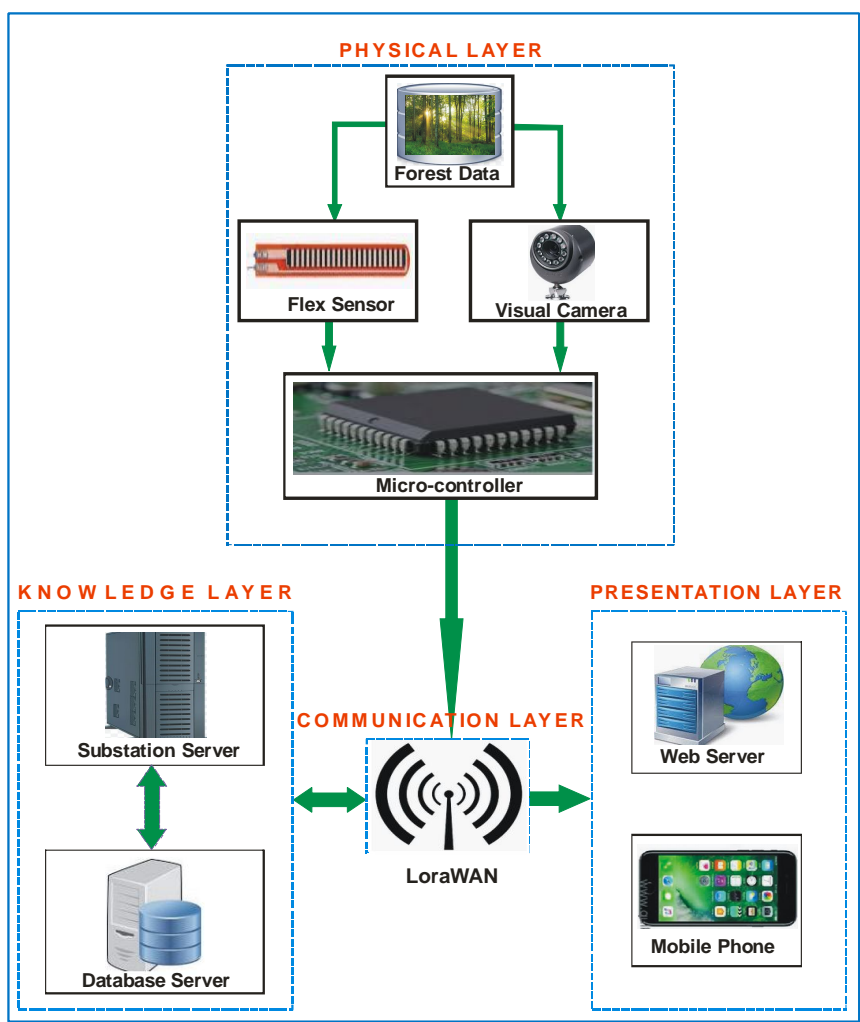

Figure 3.1: Architecture of the Deforestation Detection and Control System

\subsection{Forest Staff Records}

The table 3.1 shows a sample of forest staff records as contained in the he database.

Table 3.1: Sample of Forest Staff Record

\begin{tabular}{|c|c|c|c|c|}
\hline Name & $\begin{array}{c}\text { Staff } \\
\text { ID }\end{array}$ & Designation & Staff Tag & Picture \\
\hline $\begin{array}{c}\text { Okeke } \\
\text { Charles }\end{array}$ & OSF0 & Manager & OSF001A & \\
\hline $\begin{array}{c}\text { Kutelu } \\
\text { Daniel }\end{array}$ & OSF0 & Accountant & OSF002B & \\
\hline
\end{tabular}




\begin{tabular}{|c|c|c|c|c|}
\hline $\begin{array}{c}\text { Thomas } \\
\text { Kelvin }\end{array}$ & $\begin{array}{c}\text { OSF0 } \\
03\end{array}$ & Engineer & OSF003C \\
\hline $\begin{array}{c}\text { Arowol } \\
\text { o Kunle }\end{array}$ & $\begin{array}{c}\text { OSF0 } \\
04\end{array}$ & Guard 1 & OSF004D \\
\hline $\begin{array}{c}\text { Robert } \\
\text { Johnson }\end{array}$ & $\begin{array}{c}\text { OSF0 } \\
05\end{array}$ & Guard 2 & OSF005E \\
\hline Ovie & OSF0 & Operator1 & OSF006F \\
Nelson & 06 & & & \\
\hline Ojo & OSF0 & Operator 2 & OSF007G \\
Johnson & 07 & & & \\
\hline
\end{tabular}

\begin{tabular}{|c|c|c|c|c|}
\hline Julius & OSF0 & Horticulture & OSF008H \\
Kemi & 08 & 1 & & \\
\hline Obe & OSF0 & Horticulture & OSF009I \\
Jesikat & 09 & 2 & & \\
\hline
\end{tabular}

\subsection{Staff and Tree Mapping}

Mapping is any prescribed way of assigning to each object in one set a particular object in another (or the same) set. The mapping of each forest tree to the designated operator is shown in Table 3.2.

Table 3.2: Sample of Forest Trees and Staff Mapping

\begin{tabular}{|c|c|c|c|c|c|c|c|c|c|c|}
\hline 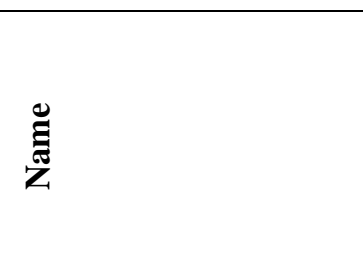 & 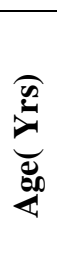 & 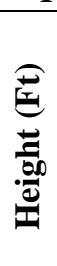 & 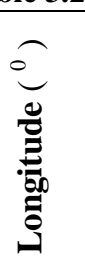 & 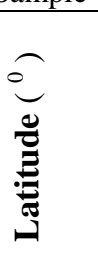 & 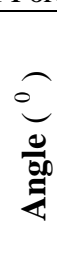 & 包 & 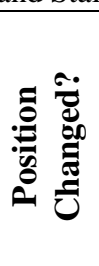 & 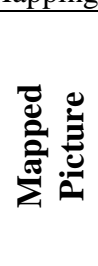 & 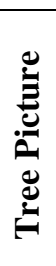 & $\begin{array}{l}\frac{8}{8} \\
\dot{\delta} \\
\frac{\tilde{\Xi}}{2} \\
\frac{\sigma}{2}\end{array}$ \\
\hline African mahogany & 6 & 15 & 31.5 & 117.5 & 90 & 001 & No & & & $\begin{array}{c}\text { OSF006F-0 } \\
01\end{array}$ \\
\hline Neem & 6 & 14 & 25.5 & 118.0 & 90 & 002 & No & & & $\begin{array}{c}\text { OSF006F-0 } \\
02\end{array}$ \\
\hline Kampalaga & 6 & 16 & 37.7 & 113.5 & 90 & 003 & No & & & $\begin{array}{c}\text { OSF006F-0 } \\
03\end{array}$ \\
\hline Natal mahogany & 5 & 27 & 43.0 & 126.0 & $\begin{array}{c}18 \\
0\end{array}$ & 014 & Yes & & & $\begin{array}{c}\text { OSF006F-0 } \\
14\end{array}$ \\
\hline Azobe & 5 & 23 & 42.0 & 122.0 & 90 & 015 & No & & & $\begin{array}{c}\text { OSF006F-0 } \\
15\end{array}$ \\
\hline Kampalaga & 5 & 24 & 44.0 & 115.0 & 90 & 016 & No & & & $\begin{array}{c}\text { OSF007G-0 } \\
16\end{array}$ \\
\hline
\end{tabular}


For proactive measure, IP Camera in the physical layer is used to take snapshots of all human entrances into the forest. This images are sent to the substation sever in the knowledge layer where the image identification module resides. The image identification module detect possible intruders into the forest as it compares the snapshot from the IP camera with those images stored in the forest database in the knowledge layer for a match. The image identification module contains image preprocessing (that is, Noise removal, Segmentation, and Feature extraction and image classification components). In image preprocessing, snapshots were smoothened to remove unwanted noise via median filter.

Thereafter, the filtered images were segmented to separate objects from their respective backgrounds using equation (4).

$q(x, y)=\left\{\begin{array}{l}1, \text { if } p(x, y) \geq T \\ 0, \text { if } p(x, y) \leq T\end{array}\right.$

where $q(x, y)$ is the output image obtained from smoothed image $p(x, y), 1$ is the object, 0 is the background, and $T$ is the threshold value that separates object from background. Hence, relevant features will be extracted from segmented images via Singular Value Decomposition (SVD) which is given as follows:

$$
X=U \Sigma V^{T}
$$

where $X$ represents the image matrix with input size $256^{*}$ 256, while $U$ and $V$ are orthogonal eigenvectors of the matrix $X X^{T}$ and $X^{T} X$ respectively, such that the columns of $U$ are the left singular vector of $\boldsymbol{X}$, and the columns of $\boldsymbol{V}$ are the right singular vector of $X$. $\Sigma$ represents the diagonal matrix of the singular values $\sigma_{i}$ presented in equation (6)

$$
\sigma_{i}=\sqrt{\lambda_{i}}
$$

$\lambda_{i}$ is the eigenvalue of $X^{T} X$ or $X X^{T}$. The singular values are used to create the feature vectors (eigenvectors) as they are sorted diagonally in a $\Sigma$ such that $\sigma_{i} \geq \sigma_{i} \geq \cdots \geq \sigma_{\min (n, n)} \geq 0$. However, the eigenvectors $x_{i} i=1,2,3, \ldots, k$ whose cumulative singular value satisfies a set threshold value were chosen to form a dataset with reduced feature subset.

The extracted features are fed into the classification module to train support vector machine (SVM) so as to distinguish intruders from non-intruders. SVM performs classification by creating decision boundaries (hyperplanes) that linearly separates the two classes.

$$
w \cdot x_{i}+b=0
$$

where $\mathrm{w}$ represents the weight vector, $b$ is the bias factor, $x_{\bar{i}}$ is instance of image in the training set. The best choice of hyperplane is the hyperplane that have the maximum margin between both classes. This is achieved by minimizing the weight vector $\|\mathrm{w}\|$ as shown in equation (8)

$$
\min _{w, b}\left(\frac{w^{T} w}{2}\right), \text { s.t. } y_{i}\left(w^{T} V_{i}+b\right) \geq 1(i=1,2, \ldots, q)
$$

where $y_{i} \in[$ inthuden, non-intruder] are class labels, and $\|w\|$ is $\left(\frac{w^{T} w}{2}\right)$.

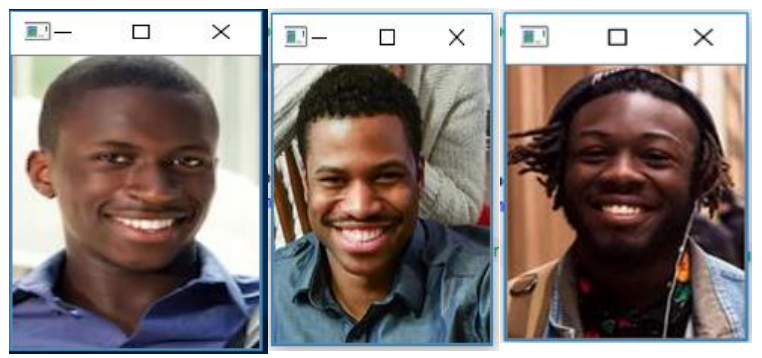

Figure 3.2: Sample of Original Images
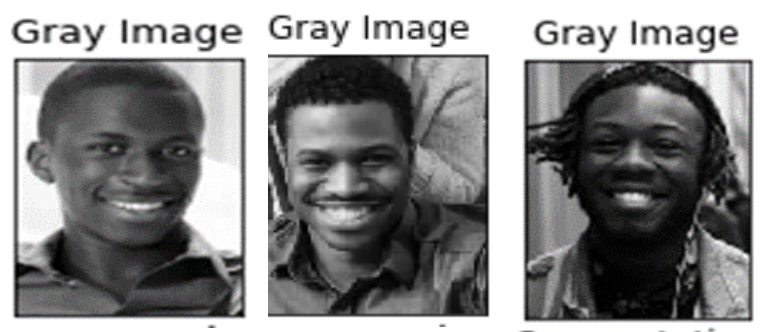

Figure 3.3: Conversion of colour images to Gray scale images

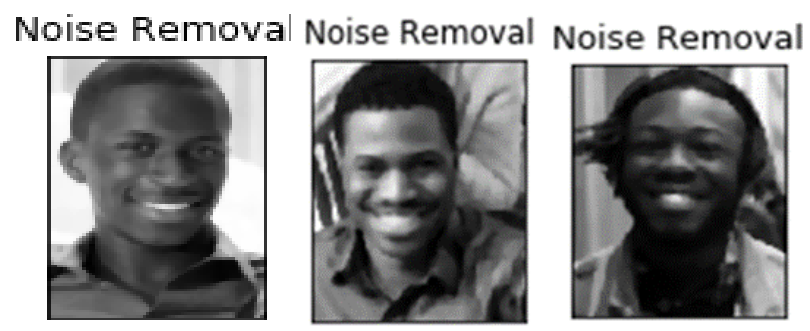

Figure 3.4: Noise Removal implementation

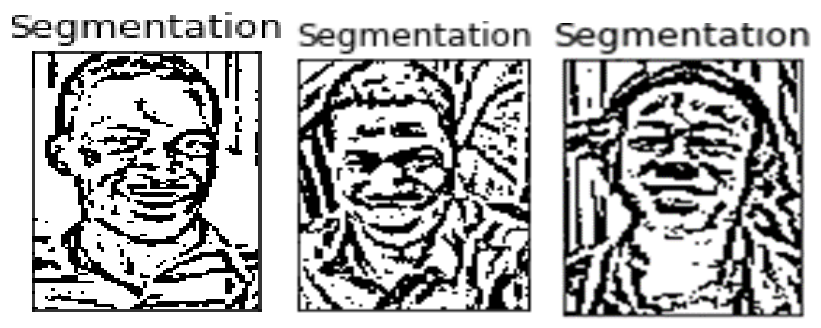

Figure 3.5: Segmentation of images
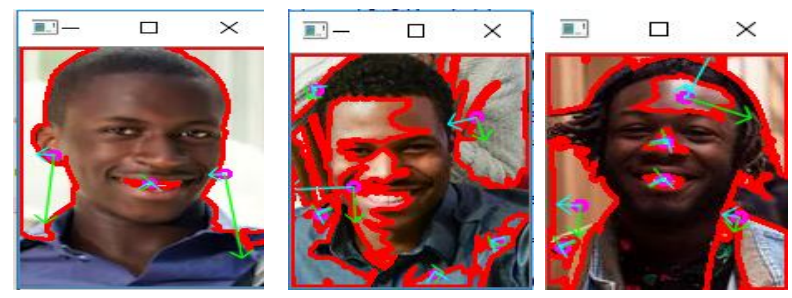

Figure 3.6: Interest points extracted by SVD 
In the case of reactive measure (that is, instance where trees are cut), the microcontroller detects the bend angle and the resistance change from the flex sensor and send deforestation alert to the web server. The sensors are positioned on selected trees to permit nodes to overlap in their sensing and communication ranges. Each sensor nodes can identify their position and spatial orientation. The nodes have the capability of localizing the source of deforestation activity within their operating range relative to their own position in order to determine if the deforestation occurs within a restricted zone. Sensors mounted on some selected trees in the forest are referred to as beacons. The beacons can detect the Angle of Arrival (AOA) information between neighbor nodes by using the triangulation approach (a method of determining the position of an object by using the bearings from known reference positions) as shown in Figure 3.7.

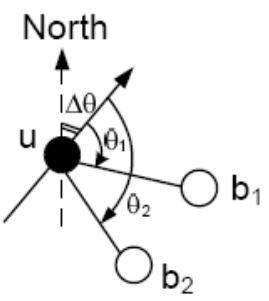

(a)

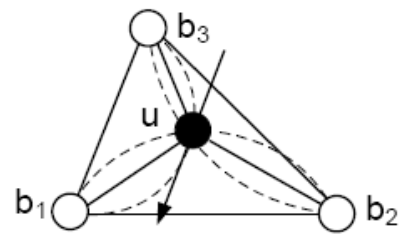

(b)
Figure 3.7: (a) Localization with orientation information; (b) Localization without orientation information.

Angles $\theta_{1}$ and $\theta_{2}$ which are measured at unknown $u$, are the relative AOAs of the signals sent from beacons $b_{1}$ and $b_{2}$ respectively. Assuming the orientation of the unknown is $\Delta \theta$, the absolute AOAs from $b_{1}$ and $b_{2}$ can be calculated by:

$\left(\theta_{i}+\Delta \theta\right)(\bmod 2 \pi)=0 \quad$ for $i=\{1,2, \ldots n\}$

where $\theta$ is the angle of arrival. Sensors deployed into the forest areas are related by:

$\left(S=\left\{S_{1}, S_{2}, \ldots . S_{n}\right\}\right.$

where $S$ is a set of connecting sensors $S_{1}, S_{2} \ldots S n$

Assuming that $n$ sensor nodes are randomly distributed over radius $R$ region and $\mathrm{A}_{\mathrm{i}}$ denote the size of area that is covered by the $i$ th placed sensor node, then total area covered by $S$ is given by:

$A=\pi R^{2}$

where $A=$ the size of the whole detected region.

The probability of barrier coverage for $S^{\prime}$ making maximum coverage fraction $R_{S}(S)$ and minimum number of working sensor nodes $|S|$ is given by:
$P_{s^{\prime}}=\left|A_{D^{\prime}}\right| /\left|A_{D}\right|$

where $S^{\prime}=$ is a subset of $S ; R_{s}=$ Region covered by minimum connecting sensor;

$\left|\mathrm{A}_{\mathrm{D}^{\prime}}\right|=$ size of the region that is covered by $S^{\prime}$ and $\left|A_{D}\right|=$ the size of region that is covered by $S$. The proportion of the number of working nodes $|S|$ to the total number of nodes $|\mathrm{S}|$ is the sub-object function $\mathrm{f}(\mathrm{x})$ of the number of working nodes as shown in equation (13)

$f(x)=R_{s}\left(S^{\prime}\right)=\left|A_{D^{\prime}}\right| /\left|A_{D}\right|$

where $\left|A_{D}\right|$ is the size of the region that is covered by $S^{\prime} ;\left|A_{D}\right|=$ size of region that is covered by $S$, and $R_{s}$ represents the region covered by minimum connecting sensor. Thus, the overall object function is given by:

$F(x)=w_{i} \cdot \frac{A_{D^{\prime}}}{A_{D}}+w_{2} \cdot\left(1-\frac{\left|S^{\prime}\right|}{|S|}\right)$

where $w_{1}$ and $w_{2}$ are the weights of sub-object function, $|S|=$ proportion of the number of working nodes, $|S|=$ total number of nodes.

Hence, information from the classification module and the microcontroller will be passed to the alert notification subsystem which uses the sub-server, webserver and the mobile system to send an alert notification of forest encroachers and deforesters to security personnel.

The flow diagram for the deforestation detection and control system is shown in Figure 3.8.

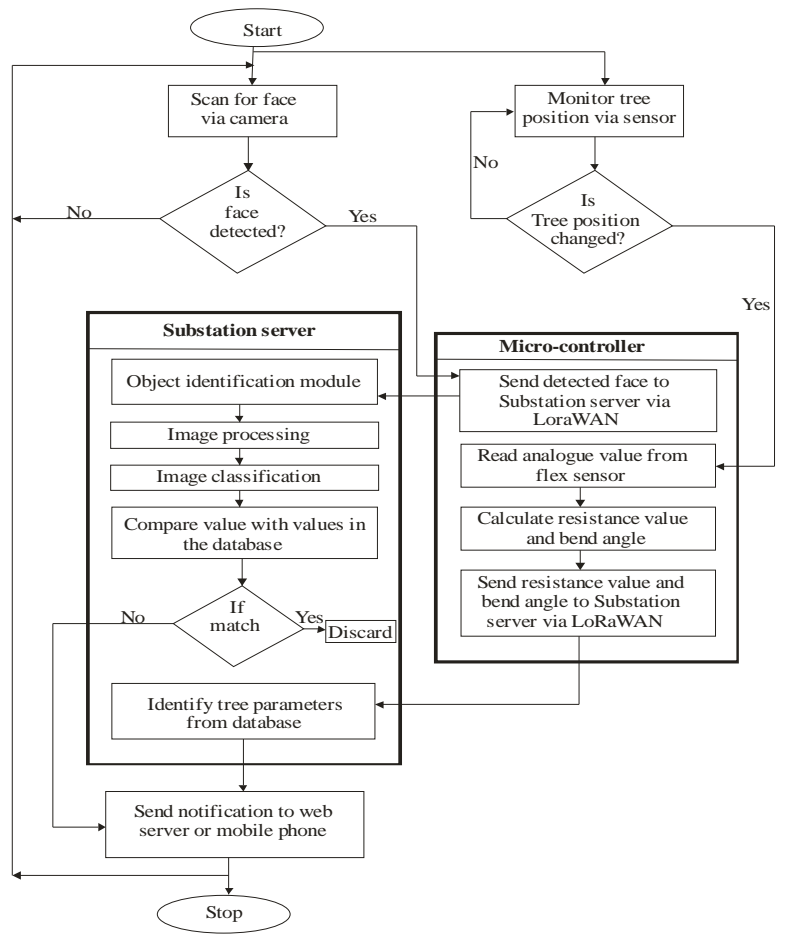

Figure 3.8: Flowchart for deforestation detection and control system 
Ibam E. O. et al., International Journal of Advanced Trends in Computer Science and Engineering, 10(3), May - June 2021, 1901 - 1913

\subsection{Class Diagram for Data Acquisition}

A class diagram demonstrates the anatomy of various classes and the relationships between the same. Figure 3.9 shows the various classes and their relationships in this design.

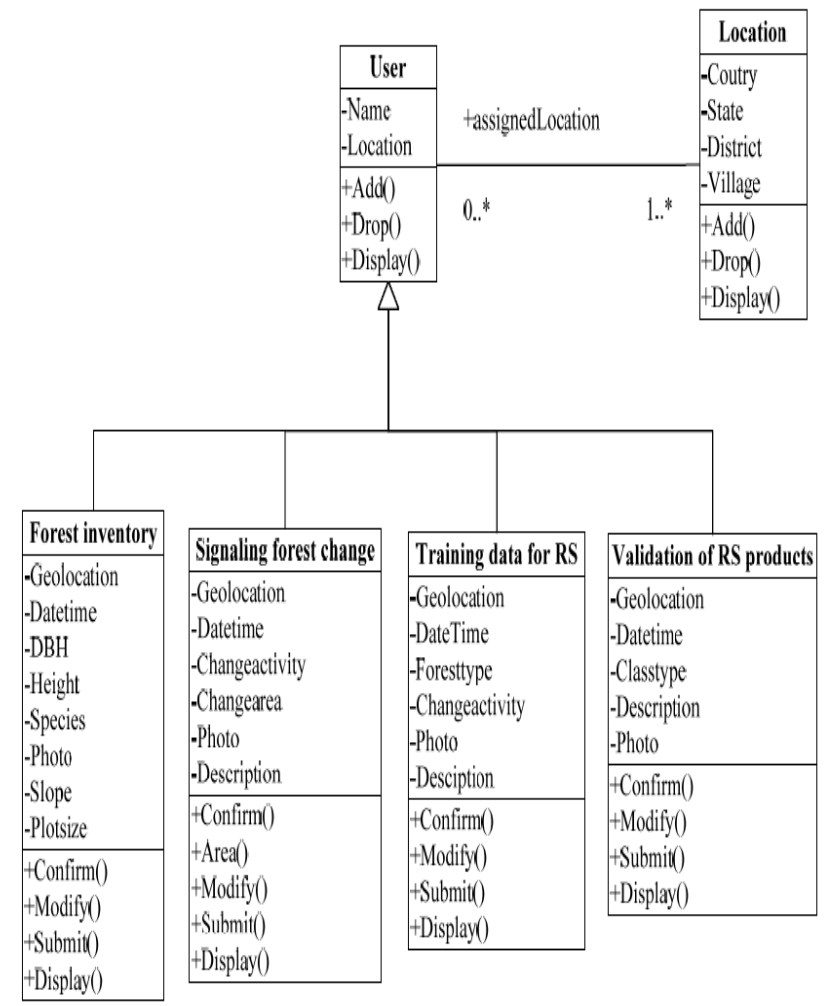

Figure 3.9: UML Class Diagram of the Deforestation Detection and Control System

\section{Fallen Tree Detection}

This is a reactive measure integrated in the system in case the intruder evades the camera. Flex sensor listens for a change in forest tree position. If a change is found in the tree position, the sensor then sends the analog value to the micro-controller.

\section{Reading analog value from sensor}

The micro-controller reads the analog value through the flex pin $\operatorname{mostly}\left(\mathrm{A}_{0}\right)$, after that the micro-controller calculate the resistance of the flex analog signal using the formula below:

Resistance

$=(1023-$ flex_value $) /\left(\right.$ flex_value $*_{\text {series }}$ s $\left.R\right)$

where variable flex_value is the analog signal read from the flex sensor and series_ $R$ is the resistance of the series resistor.

\section{Calculating bend angle}

The micro-controller at this stage, calculate the bend angle by using the formula below:

$\theta=\operatorname{map}($ flexR, straight_R, bent_R,0, 90.0) where the variable flexR stands for flex resistance, straight_R stands for the resistance of the sensor when it's straight which is about $30 \mathrm{~K} \Omega$, and bent_R stands for resistance of the sensor when it is bent which is about $70 \mathrm{~K} \Omega$. The $\operatorname{map}()$ function above uses integer math so that it will not generate fractions. Fractional remainders are truncated, and are rounded.

\section{SYSTEM IMPLEMENTATION}

\subsection{Hardware Requirement}

The hardware components used in the development of the system are Flex sensors, Forest Camera LTL Acorn 6310MG, Arduino microcontrollers, HP server, SX1301 LoRaWAN module, Mobile phone and internet network.

The server used is HP ProLiant ML10 Server which hosts the Operating System, the web applications and the DDCS application. It is a dual core processor server with 4GB RAM and 1TB SATA hard disk drive.

\subsection{Software Requirement}

The system is developed in an environment characterized by Unity 3D incorporated with $\mathrm{C \#}$ as the front end, and MySQL as the backend.

Unity $3 \mathrm{D}$ is a fully integrated development engine that provides rich out-of-the-box functionality which allows one to create amazing games and other interactive $2 \mathrm{D}$ and $3 \mathrm{D}$ content. Unity is a cross-platform game engine primarily used to develop video games and simulations for PC, consoles, mobile devices and websites. The following are major features of Unity from the technical point of view:

- Access the Components

- Events for Game Object

- Dealing with Vector Variables and Timing Variables

- $\quad$ Physics Oriented Events

Creating and Destroying Game Objects.

\subsection{Experimental Setup}

Unity 3D simulation tool was used for the experimental testbed and python was used for image processing and classification. After the image has been classified by the support vector machine modeled in python, it returns a boolean value which the $\mathrm{C} \#$ uses to start the simulation. The value returned by the python environment can either be true or false. If it is true the $\mathrm{C \#}$ program continues execution, if it is false, the execution stops. That tells the program that the object detected is not an intruder. The value returned by the python code is received by the variable "is_object_intruder" in C\#. This variable can only take a boolean variable too.

The simulation is launched by double-clicking on the application icon [Unity 2018.1f1(64 bit)] on the system desktop. The opening page is also shown in Figure 4.1. 


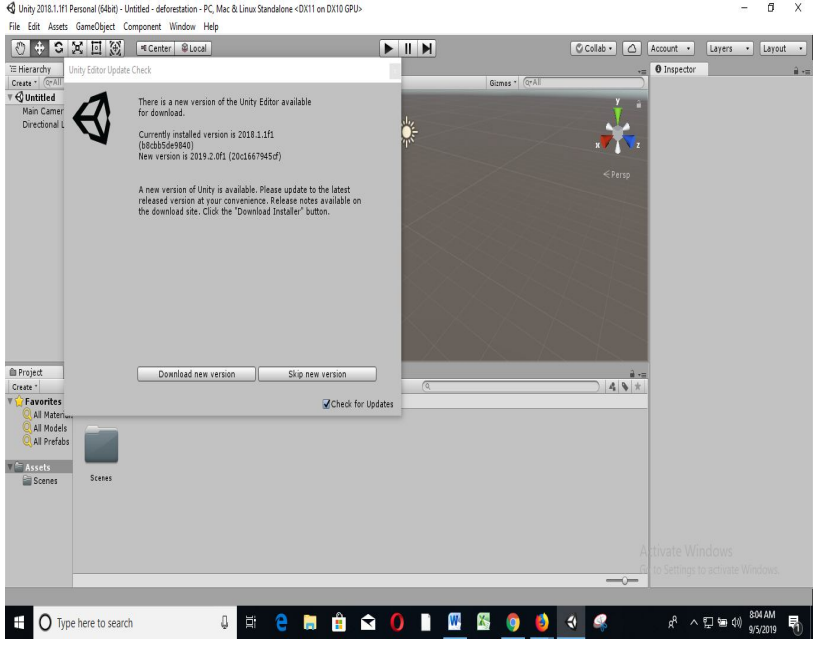

Figure 4.1: Opening Page of the Simulation

The simulation entrance scene is shown in Figure 4.2. The simulation is initialized by clicking on the run button from which the 'build object' on the screen is used to drop asset which the deforester can use while chopping down the trees.

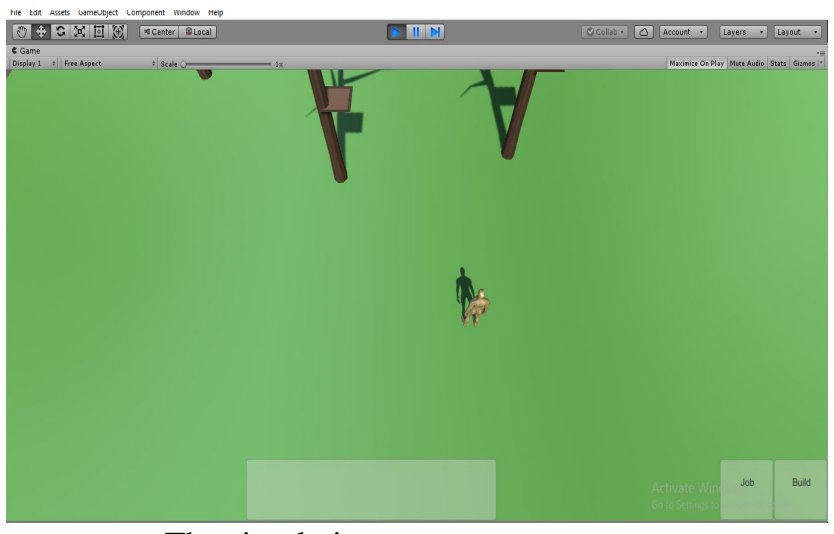

Figure 4.2: The simulation entrance scene

The job button is clicked to activate the human object (the deforester) to start action. This begins the simulation.

Figure 4.3 shows simulation character in idle position. It remains in this position until it is activated. Once activated, it interacts with the objects on the screen to find the right tool to use in the forest.

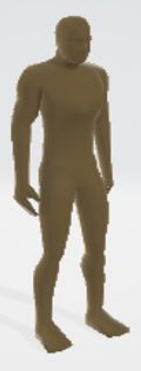

After interacting with the objects on the screen, it finally finds the right object (tool) to use in the forest, it picks it as shown in Figure 4.4. The character is in a bending position to pick and use an object.

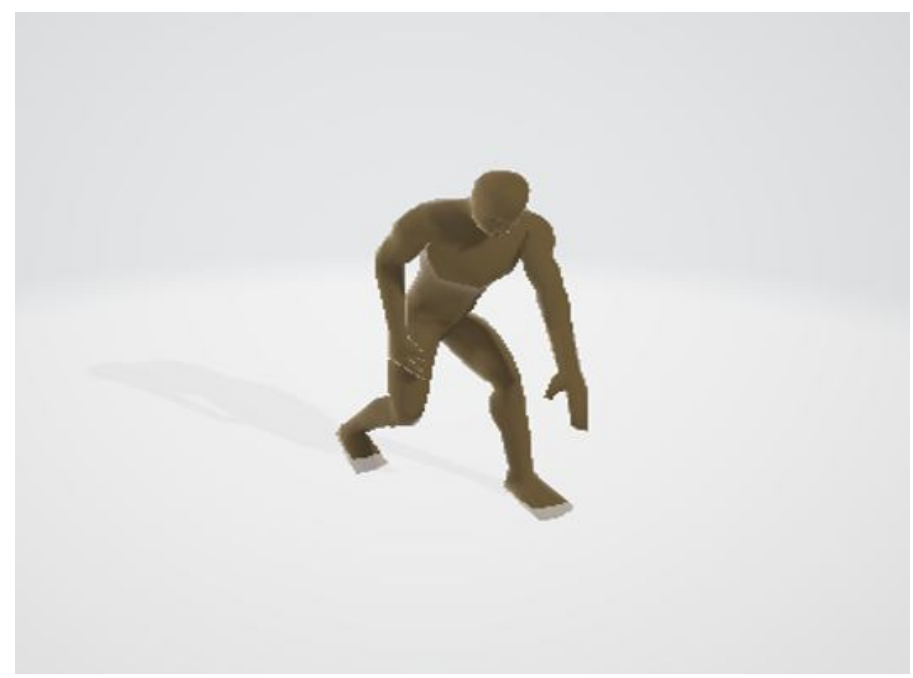

Figure 4.4: The character in a bending position to pick and use an object

As depicted by Figure 4.5 , the character is processing the wood after the right object has been found and picked, the character moves towards the tree and start cutting the trees.

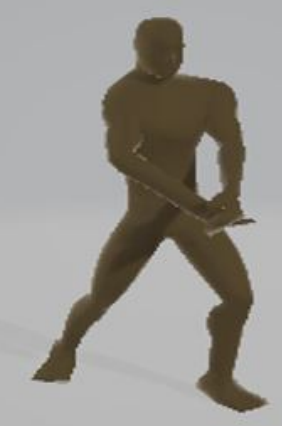

Figure 4.5: The character is processing the wood after the right object has been found

Tools available in the forest includes:

Axe: The tool is as shown in Figure 4.6, This object found on the weapon rack is the one being used to chop the tree until the tree's health is damaged beyond a certain threshold after which it falls.

Figure 4.3: Simulation character in idle position 


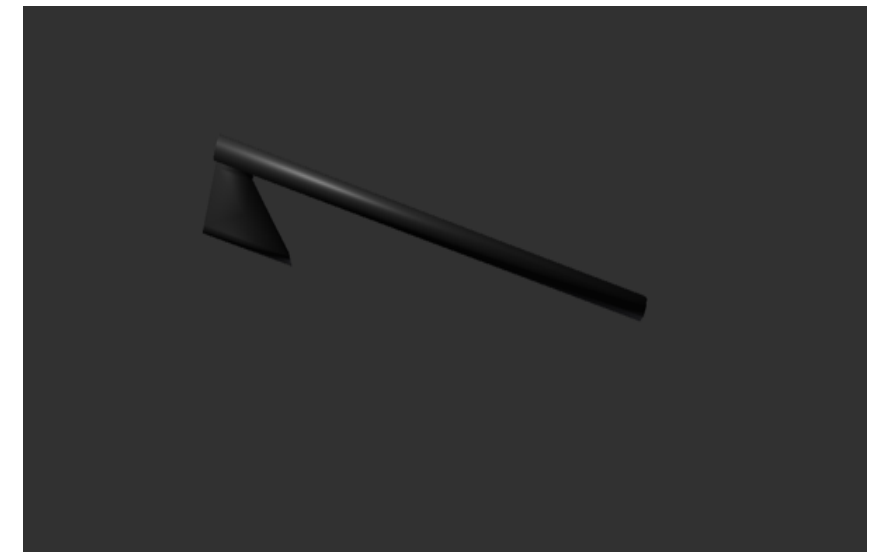

Figure 4.6: Axe- one of the available tools in the forest

In the forest, as shown by Figure 4.7, the object hanging on the trees are cameras which are used to monitor the forest area for unwanted intrusion.

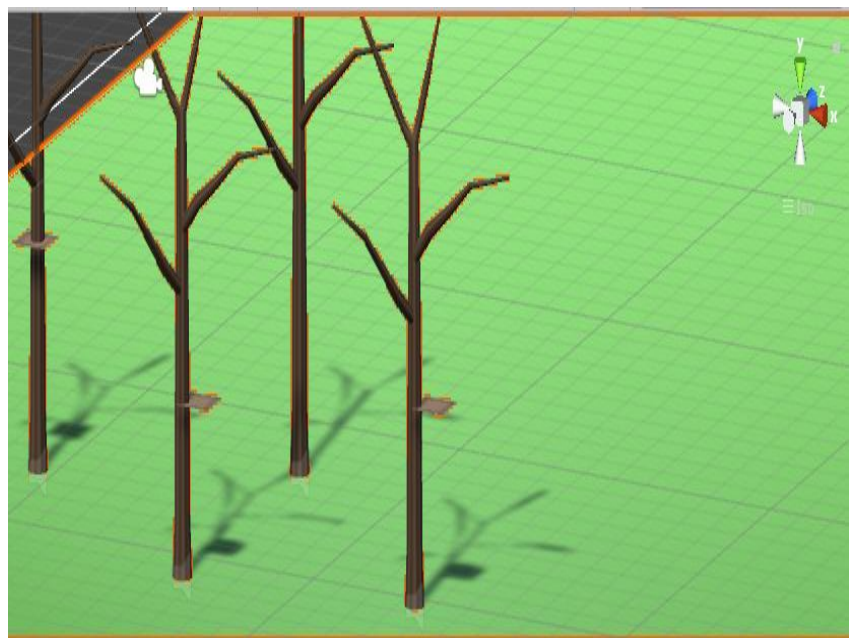

Figure 4.7: Front view of the forest area

The camera has intelligent algorithms for detecting and recognizing human. This helps to filter animals from real human beings. When human is detected, it takes a snap and communicate with the microcontroller for onward transmission to the web server. If the tree position changes or bend beyond normal, the flex sensor data send signal to the web server or mobile phone through the Lora network in the forest and the available internet network.

Also shown in Figure 4.8 is the audio sounds that are used to simulate the tree chopping and falling processes.

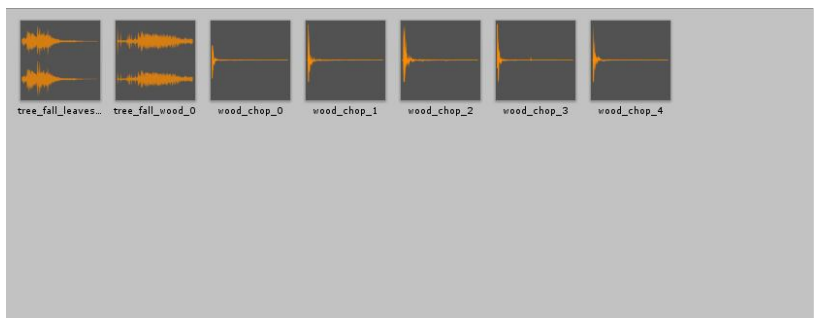

Figure 4.8: Audio sounds that are used to simulate the tree chopping and falling processes.
Shown in Figure 4.9 is the green grass used to create the environment surrounding the tree.

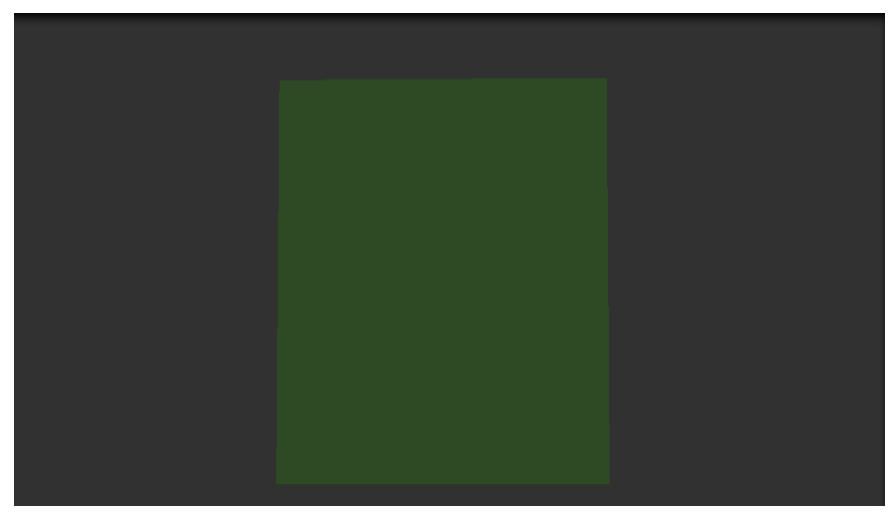

Figure 4.9: The green grass used to create the environment surrounding the tree.

Unity game engine has six parts as shown in Figure 4.10.

(i) The first is the hierarchy menu that display game object in hierarchical order. The objects on this part are objects that have been used in creating the simulation.

(ii) The second part is the inspector which displays the properties of any object selected from the object in the hierarchy menu.

(iii) The third is the project containing raw asset that has not been used in the game. Whenever a new object is to be used in the simulation, it is picked from the project part and dropped on the hierarchy part.

(iv) The forth is the scene which contains the objects dropped on the hierarchy and these objects can be manipulated from the scene to suit a particular purpose.

(v) Fifth is the game part. The game is a read only screen which displays how the game will look like after being run. Object on this screen can't be manipulated.

(vi) The sixth is the game console which is more of a debugging tool. Any error in the code is displayed on the console for proper debugging.

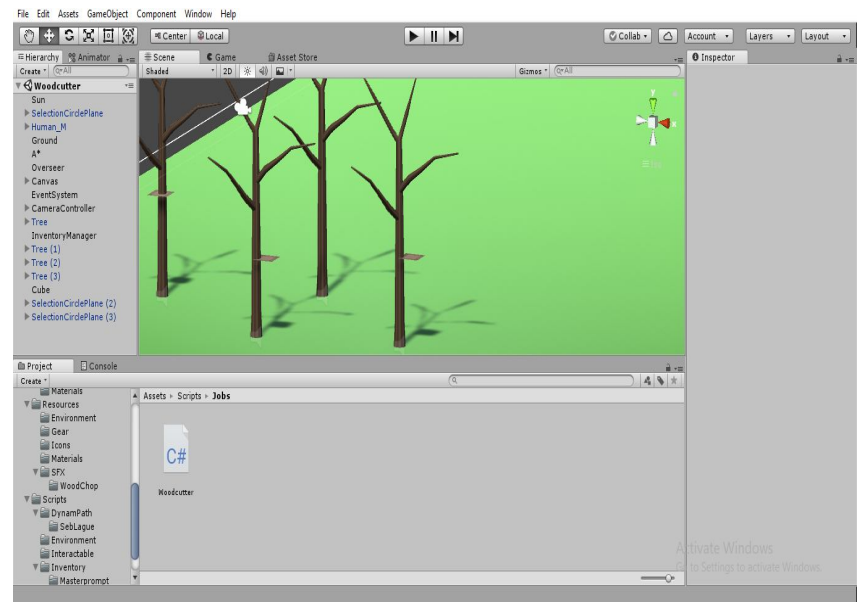

Figure 4.10: Parts of Unity Game Engine 
Ibam E. O. et al., International Journal of Advanced Trends in Computer Science and Engineering, 10(3), May - June 2021, 1901 - 1913

\section{PERFORMANCE EVALUATION}

The evaluation of the developed model is divided into two stages, namely the evaluation of detected images and tree detection.

\section{Image Detection Evaluation}

For the purpose of the research, a total number 118 images was used. In the obtained images, Seventy-two (72) images were assigned forest personnel or non-intruders, while Forty-Six (46) images were injected into the data as intruders. Table (5.1) shows the distribution of the dataset.

Table 5.1: Records of image data for the research

\begin{tabular}{|c|c|c|c|c|}
\hline \multirow{2}{*}{$\begin{array}{c}\text { Class } \\
\text { Labels }\end{array}$} & \multicolumn{5}{|c|}{ Records of image data } \\
\cline { 2 - 5 } & $\mathrm{g}$ & $\begin{array}{c}\text { Traininin } \\
\mathrm{g}\end{array}$ & $\begin{array}{c}\text { Testin } \\
\mathrm{g}\end{array}$ & $\begin{array}{c}\text { Testin } \\
\mathrm{g} \\
\%\end{array}$ \\
\hline $\begin{array}{c}\text { Legitimat } \\
\text { e users }\end{array}$ & 50 & 60.24 & 22 & 62.86 \\
\hline Intruders & 33 & 39.76 & 13 & 37.14 \\
\hline Total & $\mathbf{8 3}$ & $\mathbf{1 0 0}$ & $\mathbf{3 5}$ & $\mathbf{1 0 0}$ \\
\hline
\end{tabular}

As shown in Table (5.1), a total of 118 images were split into training and test as $70 \%$ of the images make up the training set, while the remaining $30 \%$ was for test set. In the training set 50 images are legitimate and 33 are intruders, while in the test set, 22 images are legitimate while 13 are intruders.

Hence, after the classification of images via SVM, the quality of the model was estimated on test data. This is to estimate the percentage in which the developed model correctly distinguishes forest personnel from intruders. The estimation was carried out using performance metrics such as:

Accuracy $=\frac{T P+T N}{T P+T N+F P+F N} * 100$

Specificity $=\frac{T N}{T N+F P} * 100$

Sensitivity $=\frac{T P}{T P+F N} * 100$

where Accuracy offers a general view of the system's performance as it measures the proportion of the total number of correct classifications. Specificity is the proportion of forest personnel that were correctly identified as such. Sensitivity is the proportion of intruders correctly identified as such. The parameters (TP, FP, FN, and TN) used for the computation of the aforementioned performance metrics were obtained through confusion matrix are as follows:

True Positives $(\boldsymbol{T} \boldsymbol{P})$ : the number of intruders correctly detected by the system.

False Negatives $(F N)$ : number of intruders that were incorrectly classified as forest personnel

False Positives $(F P)$ : number of forest personnel that was incorrectly classified as intruders.
True Negatives $(T N)$ : number of forest personnel that was correctly detected by the system.

Hence, Table 5.2 shows the confusion matrix of object identification subsystem on the test data.

Table 5.2: Confusion matrix of object identification subsystem

\begin{tabular}{|l|l|l|l|}
\hline \multicolumn{2}{|c|}{} & \multicolumn{2}{|l|}{ Predicted Class } \\
\cline { 3 - 4 } \multicolumn{2}{|c|}{} & $\begin{array}{l}\text { Non-Intruder } \\
\mathrm{s}\end{array}$ & Intruders \\
\hline \multirow{3}{*}{$\begin{array}{l}\text { Actual } \\
\text { Class }\end{array}$} & $\begin{array}{l}\text { Non-Intruder } \\
\mathrm{s}\end{array}$ & $T N=18$ & $F P=4$ \\
\cline { 2 - 4 } & Intruders & $F N=\mathbf{3}$ & $T P=10$ \\
\hline
\end{tabular}

Accuracy:

$$
\begin{aligned}
& \frac{T N+T P}{T W+T P+F P+F N}=\frac{13+10}{18+10+3+4}=\frac{23}{35} \%=0.8 * 100 \%=80.0 \\
& \text { Specificity }=\frac{T N}{T N+F P}=\frac{18}{18+4}=\frac{18}{22}=0.82 * 100 \%=82.0 \% \\
& \text { Sensitivity }=\frac{T P}{T P+F N}=\frac{10}{10+3}=\frac{10}{13}=0.77 * 100 \%=77.0 \%
\end{aligned}
$$

Based on the accuracy, specificity, and sensitivity results, the image identification has shown to be suitable for detection as the model recorded a general performance of $80 \%$ (accuracy), specificity $(82.0 \%)$ and sensitivity $(77.0 \%)$

\section{Tree Detection Evaluation}

The functionality and versatility of the developed system depends on its ability to meet user's specification and requirements. Major requirements of deforestation detection system are sensitivity to tree position change, and accuracy. From practical point of view, sensor base (flat state) resistance is adjusted by changing the active geometry, doubling the length will double the resistance or doubling the width halves the base resistance. Curvature radius decreases mostly in the middle of the sensor for small diameters. The sensor resistance measurement against different bending angles and voltages can be obtained by connecting a digital multimeter as shown in Table 5.3.

Table 5.3: Flex Sensor Resistance and Voltage Readings

\begin{tabular}{|l|l|l|}
\hline $\begin{array}{l}\text { Resistance in } \\
\mathrm{K} \Omega\end{array}$ & $\begin{array}{l}\text { Voltage in } \\
\text { Volts }\end{array}$ & $\begin{array}{l}\text { Angle } \\
\text { Degree }\end{array}$ \\
\hline 26.18 & 0.82 & 90 \\
\hline 40.26 & 0.67 & 75 \\
\hline 48.74 & 0.45 & 60 \\
\hline 52.92 & 0.40 & 45 \\
\hline 57.10 & 0.37 & 30 \\
\hline 60.40 & 0.32 & 15 \\
\hline 63.5 & 0.30 & 00 \\
\hline
\end{tabular}




\section{Sensor Sensitivity}

As shown in Figure 5.1, bending a flex sensor outwardly produces no change in resistance

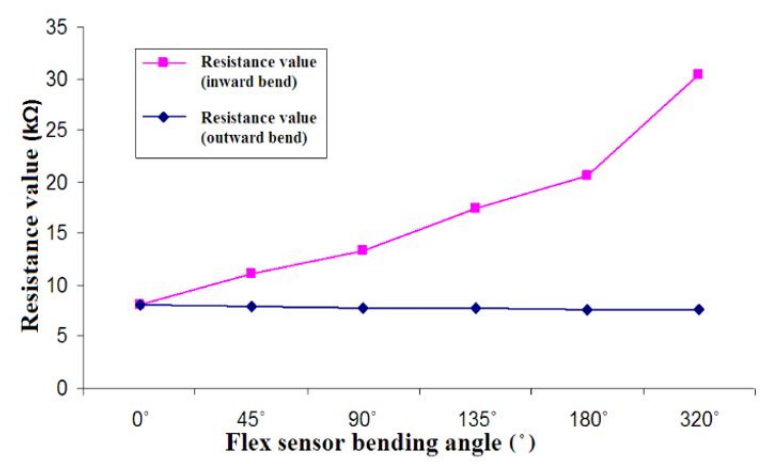

Figure 5.1: Resistance Values for Inward and Outward Bends

The result of measurement for static characteristic of a 3 and 2 -inch sensors when bent in the middle (curvature radius) for inward rotations, is reported in Figure 5.2. The nonlinearity of the characteristic implies that the resistive material must be non-isotropic (i.e non uniformity in all orientations) and must present non-uniform variation when bent.

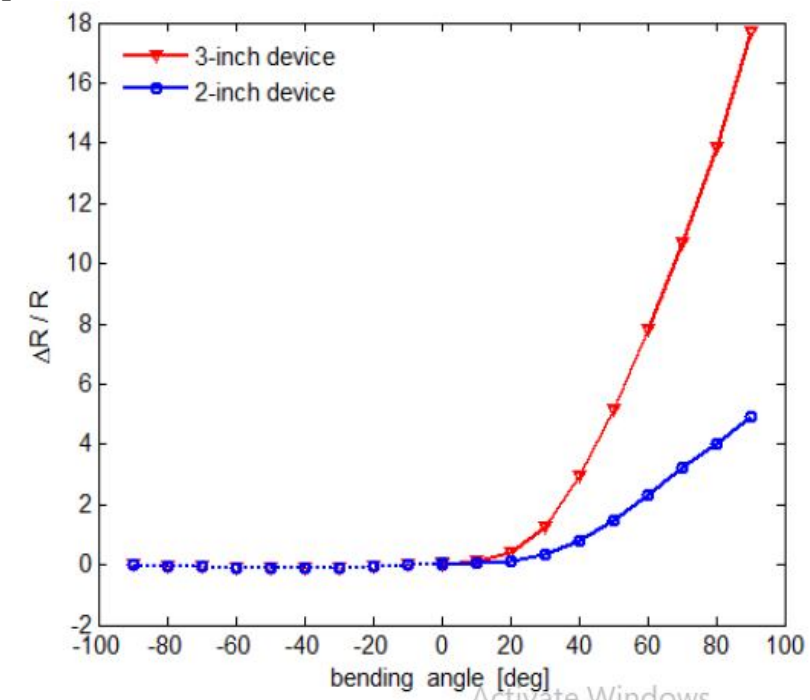

Figure 5.2: Comparative Analysis of two and three-inch flex sensors

This result cannot be exhaustive of the sensor behavior because they vary in hinge diameter (i.e a particular curvature radius) and sensor size. The behavior could be different for different radius of the hinge and different sensor size and shape.

\section{CONCLUSION}

The research carried out comprehensive review of Deforestation Detection and Control System and it highlights the effect it has on human existence of which global warming is paramount among others. More importantly, it will also assist the government to achieve their goals by putting deforesters under check and control thereby sanitizing the nation's forest industries. The transition from the traditional forest monitoring by individual forest guards to modern approach is clearly stated. Application of existing and modern forest monitoring technology in real time was adopted. This led to the design of a network framework which monitors the forest areas. The developed system consists of three subsystems; namely the database, data generation and alert notification.

The system was implemented in an environment characterized by Unity 3D incorporated with C\# programming language as the front end, and MySQL as the backend on a Windows 10 platform. Simulation using Unity 3D was used to test the functionality of the network framework. The results obtained showed that longer flex sensor produces high resistance thereby yielding better sensor sensitivity to tree position change. The cost of deployment was also lower than other existing deforestation control systems because of the use of free and open source software for its development. The research therefore established a low cost wireless sensor network framework that is able to monitor and control encroachment into forest area.

\section{Limitations of Research}

One major limitation of the research is its inability to provide mechanism that can make the system function as expected during network downtime.

\section{REFERENCES}

1. M. H. Suhaimi. A Forest Monitoring System Using Wireless sensor networks, B.S. thesis, Dept. Electri and Electron. Eng., Universiti Teknologi Petronas, Malaysia, 2007.

2. C. H. Chin, Q. Xue, and C.H. Chan. Design of a 5.8 GHz Rectenna Incorporating a New patch Antenna, IEEE Propagation Letters on Antennas and Wireless, Vol. 4, pp. 175-178, 2009.

3. F. M. Al-Turjman, H. S. Hassanein, and M. A. Ibnkahla. Connectivity Optimization for Wireless Sensor Networks Applied to Forest Monitoring, in Proc. IEEE International Conference on Communications (ICC), Dresden, Germany, 2009, pp. 1-6

4. S. Mhalsakant and S. Vaishali. Performance Analysis of Human Detection and Tracking in Changing Illumination, International Journal of Advanced Trends in Computer Science and Engineering, Vol 8, No.6, , pp. 2920- 2921, November - December 2019.

5. M. Ali, G. Yang, and R. Dougal. A New Circularly Polarized Rectenna for Wireless Power Transmission and Data Communication, IEEE Propagation Letters on Antennas and Wireless, Vol. 4, pp. 205-208, 2005.

6. T. Shi-Xiang and S. Wang. The conceptual design and simulation of mechatronic system base on UML, in Proc. 2nd International Conference on Computer Engineering and Technology, Chengdu, China, 2010, pp.V6-188-V6-192. 
7. C. A. Varuna and B. Amritha. A Wireless Sensor Network Based Habitat Monitoring for Protection of Endangered Species, International Journal of Innovative Research in Science, Engineering and Technology, Vol. 6, Issue 6, pp. 11767 - 11772, 2017.

8. D.V. Kirubaharan, Sunder A. C., S.M. Ramesh and P. Dhinakar. An Intruder Detection and Forest Fire Alert System Using Wireless Sensor Network (WSN). International Advanced Research Journal in Science, Engineering and Technology, Vol. 1, Issue 3, pp. 138 140, 2014.

9. P. Lucian and S. Gheorghe. An Energy-Efficient WSN Architecture for Illegal Deforestation Detection. International Journal of Sensors and Sensor Networks, Vol. 3, Issue 3, pp. 24-30, 2015.

10. L. Aaron and S. O. Kenneth Amazing Amazon: Detecting Deforestation in our Largest Rainforest. Accessed

http://cs231n.stanford.edu/reports/2017/pdfs/919.pdf, 2017.

11. A. Lavanya, J. Thranya and A. Jagadeesan. A Mobile Embedded Wireless Sensor Network to Prevent Deforestation. International Journal on Recent and Innovation Trends in Computing and Communication, Vol. 2, Issue 7, pp. 2045 - 2048, 2017.

12. K. R. Suguvanam, Senthil K. R., S. S. Partha, Karthick $K$. and K. S. Raj. Innovative Protection of Valuable Trees from Smuggling Using RFID and Sensors, International Journal of Innovative Research in Science, Engineering and Technology, Vol. 6, Issue 3, pp. 3836-3845, 2017. 